\title{
An inverse nodal problem for $p$-Laplacian Sturm-Liouville equation with Coulomb potential
}

\author{
Tuba Gulsen
}

Department of Mathematics, Firat University, 23119, Elazig, Turkey.

Communicated by D. Baleanu

\begin{abstract}
We deal with an inverse nodal problem for $p$-Laplacian Sturm-Liouville equation which includes Coulomb type potential function under boundary condition depends on polynomial spectral parameter. Here, we get some asymptotic formulas of eigenvalues and nodal parameters by using a suitable Prüfer substitution. Eventually, we construct Coulomb potential by using nodal lengths. (c)2017 All rights reserved.
\end{abstract}

Keywords: Inverse nodal problem, Prüfer substitution, Coulomb potential. 2010 MSC: 34A55, 34L05, 34L20.

\section{Introduction}

Inverse spectral problem consists of recovering potential function and parameters in boundary condition from the given spectral and nodal characteristics. Such problems play an important role in mathematics and have many applications in natural sciences. Inverse spectral problems are divided into two parts: inverse eigenvalue problem and inverse nodal problem. Inverse eigenvalue problem was first considered and formulated by Ambarzumian in 1929 [1]. Ambarzumian's findings can be viewed as first and vital reference in the history of inverse eigenvalue problems associated with Sturm-Liouville operators. He investigated the Schrödinger operator with Neumann boundary conditions, and found that if its spectrum consists of zero and infinitely many other square numbers, then the potential is zero. Later it became clear that the case investigated by Ambarzumian was exceptional. In general two spectra is needed to determine the potential. On the other hand, inverse nodal problem has been firstly studied by McLaughlin in 1988 [25]. The author showed that the knowledge of a dense subset of nodal points is sufficient to determine the potential function of Sturm-Liouville problem up to a constant. Also, some numerical results about this problem were given in [17]. Nowadays, many authors have obtained some interesting results about inverse nodal problems for different type operators [6, 8, 17, 19, 21-24, 26, 28, 35, 36].

Because of its importance, we have decided to give some more general results and solve an inverse nodal problem for the Sturm-Liouville equation with Coulomb potential that we have defined in $p$ Laplacian form. Therefore, we have generalized classical problem to $p$-Laplacian case. $p$-Laplacian type

Email address: tubagulsen87@hotmail.com (Tuba Gulsen)

doi:10.22436/jnsa.010.10.24 
equations have been considered by many authors for different equations and conditions $[5,11,12,15,18$, $20,30,31]$. But our study will be the first according to our researches. Because results in literature about this problem in classical case will be special case of our study when $p=2$.

Consider the following p-Laplacian Sturm-Liouville eigenvalue problem with Coulomb potential

$$
-\left(y^{\prime(p-1)}\right)^{\prime}=(p-1)\left(\lambda-\frac{\delta}{x}-q_{m}(x)\right) y^{(p-1)}, \quad 1 \leqslant x \leqslant a,
$$

with boundary conditions

$$
\begin{aligned}
& y(1)=0, \quad y^{\prime}(1)=1 \\
& y^{\prime}(a)+f(\lambda) y(a)=0,
\end{aligned}
$$

where $p>1$,

$$
f(\lambda)=b_{1} \sqrt{\lambda}+b_{2}(\sqrt{\lambda})^{2}+\cdots+b_{m}(\sqrt{\lambda})^{m}, \quad b_{i} \in \mathbb{R}, \quad b_{m} \neq 0, \quad m \in \mathbb{Z}^{+},
$$

$\lambda$ is a spectral parameter, $\delta, a \in \mathbb{R}(a>1)$ and $y^{(p-1)}=|y|^{(p-2)} y$. Throughout this study, we assume that $q_{m}(x)$ are real-valued $C[1, a]$-functions for each $m \in \mathbb{Z}^{+}$and $y(x, \lambda)$ denotes the eigenfunction of the problem (1.1) and (1.2). Equation (1.1) becomes well-known Sturm-Liouville equation with Coulomb potential for $p=2$ which has been studied by various authors (see $[2-4,9,10,16]$ ).

The idea of inverse eigenvalue problems with an eigenparameter in the boundary conditions is of great interest to many problems of mathematical physics and mechanics. These type problems have many physical applications. For instance, Sturm-Liouville equation including spectral parameter in boundary conditions arises in heat and one-dimensional wave equation by seperation of variables. There are many studies on these type problems in the literature (see $[7,13,14,27,29,32,33])$.

The set $X_{n}=\left\{x_{j, m}^{n}\right\}_{j=1}^{n-1}$ of the eigenfunction $y_{n, m}(x)$ corresponding to $\lambda_{n, m}$ is called the set of nodal points, and $l_{j, m}^{n}=x_{j+1, m}^{n}-x_{j, m}^{n}$ is referred to the nodal length of $y_{n, m} \cdot y_{n, m}\left(x, \lambda_{m}\right)$ has exactly $n-1$ nodal points in $(1, a)$ as

$$
0=x_{0, m}^{(n)}<x_{1, m}^{(n)}<\cdots<x_{n-1, m}^{(n)}<x_{n, m}^{(n)}=1 .
$$

To explain our results, we need to introduce generalized sine function $S_{p}$ which is the solution of the following initial value problem

$$
\begin{gathered}
-\left(S_{p}^{\prime(p-1)}\right)^{\prime}=(p-1) S_{p}^{(p-1)}, \\
S_{p}(0)=0, \quad S_{p}^{\prime}(0)=1,
\end{gathered}
$$

where $S_{p}$ and $S_{p}^{\prime}$ are periodic functions which satisfy the identity

$$
\left|S_{p}(x)\right|^{p}+\left|S_{p}^{\prime}(x)\right|^{p}=1
$$

for any $x \in \mathbb{R}$. These functions are $p$-analogues of classical sine and cosine functions. It is well-known that

$$
\hat{\pi}=\frac{2 \pi}{p \sin \left(\frac{\pi}{p}\right)},
$$

is the first zero of $S_{p}$ in positive axis (see [11]). Note that the following lemma is crucial in our results and was given in [11].

Lemma 1.1 ([11]).

(a) For $S_{p}^{\prime} \neq 0$,

$$
\left(S_{p}^{\prime}\right)^{\prime}=-\left|\frac{S_{p}}{S_{p}^{\prime}}\right|^{p-2} S_{p} .
$$


(b)

$$
\left(S_{p} S_{p}^{\prime(p-1)}\right)^{\prime}=\left|S_{p}^{\prime}\right|^{p}-(p-1) S_{p}^{p}=1-p\left|S_{p}\right|^{p}=(1-p)+p\left|S_{p}^{\prime}\right|^{p} .
$$

Using $S_{p}(x)$ and $S_{p}^{\prime}(x)$, the generalized tangent function $T_{p}(x)$ can be defined as [11]

$$
\mathrm{T}_{\mathrm{p}}(\mathrm{x})=\frac{\mathrm{S}_{\mathrm{p}}(\mathrm{x})}{\mathrm{S}_{\mathrm{p}}^{\prime}(\mathrm{x})}, \quad \text { for } \mathrm{x} \neq\left(\mathrm{k}+\frac{1}{2}\right) \hat{\pi}
$$

The remaining part of this study is organized as follows: in Section 2, we give some asymptotic formulas for eigenvalues and nodal parameters for $p$-Laplacian Sturm-Liouville eigenvalue problem (1.1) and (1.2) by using modified Prüfer substitution. In Section 3, we give a reconstruction formula for Coulomb potential. Finally, we give some conclusions in Section 4.

\section{Asymptotics of some eigenparameters}

In this section, we present some important results for problem (1.1) and (1.2). One of them is Prüfer's transformation which is one of the most powerful methods for solution of inverse problem. Prüfer's transformation for a nonzero solution of $y$ for (1.1) takes the form

or

$$
\begin{aligned}
y(x) & =R(x) S_{p}\left(\lambda^{1 / p} \theta(x, \lambda)\right), \\
y^{\prime}(x) & =\delta \lambda^{1 / p} R(x) S_{p}^{\prime}\left(\lambda^{1 / p} \theta(x, \lambda)\right),
\end{aligned}
$$

$$
\frac{y^{\prime}(x)}{y(x)}=\delta \lambda^{1 / p} \frac{S_{p}^{\prime}\left(\lambda^{1 / p} \theta(x, \lambda)\right)}{S_{p}\left(\lambda^{1 / p} \theta(x, \lambda)\right)}
$$

where $R(x)$ is amplitude and $\theta(x)$ is Prüfer variable [34]. Standard manipulations yield

$$
\theta^{\prime}(x, \lambda)=\delta+\left(-\delta+\delta^{1-p}-\frac{\delta^{1-p}}{\lambda} q_{m}^{c}(x)\right) S_{p}^{p}\left(\lambda^{1 / p} \theta(x, \lambda)\right),
$$

where $q_{m}^{C}(x)=\frac{\delta}{x}+q_{m}(x)$.

Lemma 2.1 ([31]). Define $\theta(x, \lambda)$ as in $(2.1)$ and $\phi(x)=S_{p}^{p}\left(\lambda^{1 / p} \theta(x, \lambda)\right)-\frac{1}{p}$. Then, for any $g \in L^{1}(1, a)$

$$
\int_{1}^{a} \phi(x) g(x) d x=0
$$

Theorem 2.2. The eigenvalues $\lambda_{n, m}$ of the problem (1.1) and (1.2) have the form

$$
\lambda_{n, 1}^{1 / p}=\frac{n \hat{\pi}}{\tilde{\delta}(a-1)}-\frac{\delta(a-1)^{\frac{p-4}{2}}}{b_{1} \tilde{\delta}^{\frac{4-p}{2}}(n \hat{\pi})^{\frac{p-2}{2}}}+\frac{\delta^{1-p}(a-1)^{p-2}}{p \tilde{\delta}^{2-p}(n \hat{\pi})^{p-1}} \int_{1}^{a} q_{1}^{C}(x) d x+O\left(\frac{1}{n^{p-1}}\right)
$$

for $\mathrm{m}=1$, and

$$
\begin{aligned}
\lambda_{n, m}^{1 / p}= & \frac{n \hat{\pi}}{\tilde{\delta}(a-1)}-\frac{\delta(a-1)^{\frac{m p-4}{2}}}{\tilde{\delta}^{\frac{4-m p}{2}}\left(b_{1} \tilde{\delta}^{\frac{m p-p}{2}}(n \hat{\pi})^{\frac{p-2}{2}}(a-1)^{\frac{m p-p}{2}}+\cdots+b_{m}(n \hat{\pi})^{\frac{m p-2}{2}}\right)} \\
& +\frac{\delta^{1-p}(a-1)^{p-2}}{p \tilde{\delta}^{2-p}(n \hat{\pi})^{p-1}} \int_{1}^{a} q_{m}^{c}(x) d x+O\left(\frac{1}{n^{2 p-1}}\right)
\end{aligned}
$$

for $\mathrm{m} \geqslant 2$ as $\mathrm{n} \rightarrow \infty$, respectively, where $\tilde{\delta}=\delta\left(1-\frac{1}{\mathrm{p}}+\frac{1}{\mathrm{p} \delta^{\mathrm{p}}}\right)$. 
Proof. Let $\theta(1, \lambda)=0$ for problem (1.1) and (1.2). Integrating both sides of (2.2) with respect to $x$ from 1 to $a$, we get

$$
\theta(a, \lambda)=\delta(a-1)+\int_{1}^{a}\left(-\delta+\delta^{1-p}-\frac{\delta^{1-p}}{\lambda} q_{m}^{c}(x)\right) S_{p}^{p}\left(\lambda^{1 / p} \theta(x, \lambda)\right) d x .
$$

By Lemma 2.1,

$$
\int_{1}^{a} q_{m}^{c}(x)\left\{S_{p}^{p}\left(\lambda^{1 / p} \theta(x, \lambda)\right)-\frac{1}{p}\right\} d x=o(1), \text { as } n \rightarrow \infty
$$

Hence, we obtain

$$
\theta(a, \lambda)=\tilde{\delta}(a-1)-\frac{\delta^{1-p}}{p \lambda} \int_{1}^{a} q_{m}^{c}(x) d x+O\left(\frac{1}{\lambda}\right) .
$$

Let $\lambda_{n, m}$ be the eigenvalues of the problem (1.1)-(1.2). For $m=1$, by (1.2), we have

$$
\delta \lambda_{n, 1}^{1 / p} R(a) S_{p}^{\prime}\left(\lambda_{n, 1}^{1 / p} \theta\left(a, \lambda_{n, 1}\right)\right)+b_{1} \sqrt{\lambda_{n, 1}} R(a) S_{p}\left(\lambda_{n, 1}^{1 / p} \theta\left(a, \lambda_{n, 1}\right)\right)=0,
$$

or

$$
-\frac{\delta \lambda_{n, 1}^{\frac{1}{p}-\frac{1}{2}}}{b_{1}}=\frac{S_{p}\left(\lambda_{n, 1}^{1 / p} \theta\left(a, \lambda_{n, 1}\right)\right)}{S_{p}^{\prime}\left(\lambda_{n, 1}^{1 / p} \theta\left(a, \lambda_{n, 1}\right)\right)}=T_{p}\left(\lambda_{n, 1}^{1 / p} \theta\left(a, \lambda_{n, 1}\right)\right) .
$$

For sufficiently large $n$, it follows from (2.6) that

$$
\lambda_{n, 1}^{1 / p} \theta\left(a, \lambda_{n, 1}\right)=T_{p}^{-1}\left(-\frac{\delta \lambda_{n, 1}^{\frac{1}{p}-\frac{1}{2}}}{b_{1}}\right)=n \hat{\pi}-\frac{\delta \lambda_{n, 1}^{\frac{1}{p}-\frac{1}{2}}}{b_{1}}+o\left(\lambda_{n, 1}^{\frac{2}{p}-1}\right) .
$$

By considering (2.5) and (2.6) together, we get

$$
\lambda_{n, 1}^{1 / p}=\frac{n \hat{\pi}}{\tilde{\delta}(a-1)}-\frac{\delta(a-1)^{\frac{p-4}{2}}}{b_{1} \tilde{\delta}^{\frac{4-p}{2}}(n \hat{\pi})^{\frac{p-2}{2}}}+\frac{\delta^{1-p}(a-1)^{p-2}}{p^{2} \tilde{\delta}^{2-p}(n \hat{\pi})^{p-1}} \int_{1}^{a} q_{1}^{C}(x) d x+O\left(\frac{1}{n^{p-1}}\right) .
$$

For $m \geqslant 2$, by (1.2), using the same process as in $m=1$, we can easily obtain the following formula

$$
\delta \lambda_{n, m}^{1 / p} R(a) S_{p}^{\prime}\left(\lambda_{n, m}^{1 / p} \theta\left(a, \lambda_{n, m}\right)\right)+\left(b_{1} \sqrt{\lambda_{n, m}}+\cdots+b_{m}\left(\sqrt{\lambda_{n, m}}\right)^{m}\right) R(a) S_{p}\left(\lambda_{n, m}^{1 / p} \theta\left(a, \lambda_{n, m}\right)\right)=0
$$

or

$$
-\frac{\delta \lambda_{n, m}^{\frac{1}{p}}}{b_{1} \sqrt{\lambda_{n, m}}+\cdots+b_{m}\left(\sqrt{\lambda_{n, m}}\right)^{m}}=\frac{S_{p}\left(\lambda_{n, m}^{1 / p} \theta\left(a, \lambda_{n, m}\right)\right)}{S_{p}^{\prime}\left(\lambda_{n, m}^{1 / p} \theta\left(a, \lambda_{n, m}\right)\right)}=T_{p}\left(\lambda_{n, m}^{1 / p} \theta\left(a, \lambda_{n, m}\right)\right),
$$

by considering (2.5) and (2.7) together, we deduce that

$$
\begin{aligned}
\lambda_{n, m}^{1 / p}= & \frac{n \hat{\pi}}{\tilde{\delta}(a-1)}-\frac{\delta(a-1)^{\frac{m p-4}{2}}}{\tilde{\delta}^{\frac{4-m p}{2}}\left(b_{1} \tilde{\delta}^{\frac{m p-p}{2}}(n \hat{\pi})^{\frac{p-2}{2}}(a-1)^{\frac{m p-p}{2}}+\cdots+b_{m}(n \hat{\pi})^{\frac{m p-2}{2}}\right)} \\
& +\frac{\delta^{1-p}(a-1)^{p-2}}{p \tilde{\delta}^{2-p}(n \hat{\pi})^{p-1}} \int_{1}^{a} q_{m}^{C}(x) d x+O\left(\frac{1}{n^{2 p-1}}\right) .
\end{aligned}
$$


Theorem 2.3. Asymptotic formula of the nodal points for problem (1.1) and (1.2) satisfies

$$
\begin{aligned}
& x_{j, 1}^{n}=1+\frac{j \tilde{\delta}(a-1)}{\delta n}-\frac{j \tilde{\delta}^{p / 2}(a-1)^{p / 2}}{b_{1} n^{\frac{p+2}{2}} \hat{\pi}^{\frac{p}{2}}}+\frac{j \tilde{\delta}^{p}(a-1)^{p}}{p \delta^{p} n^{p+1} \hat{\pi}^{p}} \int_{1}^{a} q_{1}^{c}(t) d t \\
& +\int_{1}^{x_{j, 1}^{\mathfrak{n}}}\left(1-\frac{1}{\delta^{\mathfrak{p}}}+\frac{\tilde{\delta}^{p}(\mathrm{a}-1)^{\mathfrak{p}}}{\delta^{\mathfrak{p}}(\mathrm{n} \hat{\pi})^{\mathfrak{p}}} \mathrm{q}_{1}^{\mathrm{C}}(\mathrm{t})\right) S_{\mathfrak{p}}^{\mathfrak{p}} \mathrm{dt}+\mathrm{O}\left(\frac{j}{n^{\mathfrak{p}+1}}\right)
\end{aligned}
$$

and

$$
\begin{aligned}
x_{j, m}^{\mathfrak{n}}= & 1+\frac{j \tilde{\delta}(a-1)}{\delta n}-\frac{j \tilde{\delta}^{m p / 2}(a-1)^{m p / 2}}{b_{1} n^{\frac{p+2}{2}} \hat{\pi}^{\frac{p}{2}}(a-1)^{\frac{m p-p}{2}} \tilde{\delta}^{\frac{m p-p}{2}}+\cdots+b_{m} n^{\frac{m p+2}{2}} \hat{\pi}^{\frac{m p}{2}}}+\frac{j \tilde{\delta}^{p}(a-1)^{p}}{p \delta^{p} n^{p+1} \hat{\pi}^{p}} \int_{1}^{a} q_{m}^{C}(t) d t \\
& +\int_{1}^{x_{j, m}^{n}}\left(1-\frac{1}{\delta^{p}}+\frac{\tilde{\delta}^{p}(a-1)^{p}}{\delta^{p}(n \pi)^{p}} q_{m}^{C}(t)\right) S_{p}^{p} d t+O\left(\frac{j}{n^{2 p+1}}\right)
\end{aligned}
$$

for $\mathrm{m}=1$ and $\mathrm{m} \geqslant 2$, respectively as $\mathrm{n} \rightarrow \infty$.

Proof. Integrating (2.2) from 1 to $x_{j, m}^{n}$ and letting $\theta\left(x_{j, m}^{n}, \lambda\right)=\frac{j \hat{\pi}}{\lambda_{n, m}^{1 / p}}$, we have

$$
x_{j, m}^{n}=1+\frac{j \hat{\pi}}{\delta \lambda_{n, m}^{1 / p}}+\int_{1}^{x_{j, m}^{n}}\left(1-\frac{1}{\delta^{p}}+\frac{1}{\delta^{p} \lambda_{n, m}} q_{m}^{c}(t)\right) S_{p}^{p} d t
$$

For $m=1$, from the formula (2.3), we deduce

$$
\frac{1}{\lambda_{n, 1}^{1 / p}}=\frac{\tilde{\delta}(a-1)}{n \hat{\pi}}-\frac{\delta(a-1)^{p / 2} \tilde{\delta}^{p / 2}}{b_{1}(n \hat{\pi})^{\frac{p+2}{2}}}+\frac{\delta^{1-p}(a-1)^{p} \tilde{\delta}^{p}}{p(n \hat{\pi})^{p+1}} \int_{1}^{a} q_{1}^{C}(x) d x+O\left(\frac{1}{n^{p+1}}\right),
$$

and therefore we obtain formula (2.8) by using equations (2.10) and (2.11).

For $m \geqslant 2$, from the formula (2.4), we can easily obtain the following formula

$$
\begin{aligned}
\frac{1}{\lambda_{n, m}^{1 / p}}= & \frac{\tilde{\delta}(a-1)}{n \hat{\pi}}-\frac{\delta \tilde{\delta}^{m p / 2}(a-1)^{m p / 2}}{b_{1}(n \hat{\pi})^{\frac{p+2}{2}}(a-1)^{\frac{m p-p}{2}} \tilde{\delta}^{\frac{m p-p}{2}}+\cdots+b_{m}(n \hat{\pi})^{\frac{m p+2}{2}}} \\
& +\frac{\delta^{1-p}(a-1)^{p} \tilde{\delta}^{p}}{p(n \hat{\pi})^{p+1}} \int_{1}^{a} q_{m}^{C}(x) d x+O\left(\frac{1}{n^{2 p+1}}\right)
\end{aligned}
$$

and we get the formula (2.9) by using equations (2.10) and (2.12).

Theorem 2.4. Asymptotic formula of the nodal lengths for the problem (1.1) and (1.2) satisfies

$$
\begin{aligned}
& l_{j, 1}^{n}=\frac{\tilde{\delta}(a-1)}{n \hat{\pi}}-\frac{\tilde{\delta}^{p / 2}(a-1)^{p / 2}}{b_{1} n^{\frac{p+2}{2}} \hat{\pi}^{\frac{p}{2}}}+\frac{\tilde{\delta}^{p}(a-1)^{p}}{p \delta^{p} n^{p+1} \hat{\pi}^{p}} \int_{1}^{a} q_{1}^{C}(t) d t \\
& +\int_{\chi_{j, 1}^{\mathfrak{n}}}^{x_{j+1,1}^{\mathfrak{n}}}\left(1-\frac{1}{\delta^{p}}+\frac{\tilde{\delta}^{p}(a-1)^{p}}{\delta^{p}(n \hat{\pi})^{p}} q_{1}^{C}(t)\right) S_{p}^{p} d t+O\left(\frac{1}{n^{p+1}}\right),
\end{aligned}
$$


and

$$
\begin{aligned}
l_{j, m}^{n}= & \frac{\tilde{\delta}(a-1)}{n \hat{\pi}}-\frac{\tilde{\delta}^{m p / 2}(a-1)^{m p / 2}}{b_{1} n^{\frac{p+2}{2}} \hat{\pi}^{\frac{p}{2}}(a-1)^{\frac{m p-p}{2}} \tilde{\delta}^{\frac{m p-p}{2}}+\cdots+b_{m} n^{\frac{m p+2}{2}} \hat{\pi}^{\frac{m p}{2}}}+\frac{\tilde{\delta}^{p}(a-1)^{p}}{p^{p} n^{p}+1 \hat{\pi}^{p}} \int_{1}^{a} q_{m}^{c}(t) d t \\
& +\int_{x_{j, m}^{n}}^{x_{j+1, m}^{n}}\left(1-\frac{1}{\delta^{p}}+\frac{\tilde{\delta}^{p}(a-1)^{p}}{\delta^{p}(n \hat{\pi})^{p}} q_{m}^{C}(t)\right) S_{p}^{p} d t+O\left(\frac{1}{n^{2 p+1}}\right)
\end{aligned}
$$

for $\mathrm{m}=1$ and $\mathrm{m} \geqslant 2$, respectively as $\mathrm{n} \rightarrow \infty$.

Proof. For large $n \in \mathbb{N}$, integrating (2.2) in $\left[x_{j, m}^{n}, x_{j+1, m}^{n}\right]$ and using the definition of nodal lengths, we have

$$
\begin{aligned}
\frac{\hat{\pi}}{\delta \lambda_{n, m}^{1 / p}}= & \left(x_{j+1, m}^{n}-x_{j, m}^{n}\right)-\frac{1}{p} \int_{x_{j, m}^{n}}^{x_{j+1, m}^{n}}\left(1-\frac{1}{\delta^{p}}+\frac{1}{\delta^{p} \lambda_{n, m}} q_{m}^{c}(t)\right) d t \\
& -\int_{x_{j, m}^{n}}^{x_{j+1, m}^{n}}\left(1-\frac{1}{\delta^{p}}+\frac{1}{\delta^{p} \lambda_{n, m}} q_{m}^{c}(t)\right)\left(S_{p}^{p}-\frac{1}{p}\right) d t
\end{aligned}
$$

or

$$
l_{j, m}^{n}=\frac{\hat{\pi}}{\delta \lambda_{n, m}^{1 / p}}+\frac{1}{p} \int_{x_{j, m}^{n}}^{x_{j+1, m}^{n}}\left(1-\frac{1}{\delta^{p}}+\frac{1}{\delta^{p} \lambda_{n, m}} q_{m}^{c}(t)\right) d t+O\left(\frac{1}{\lambda_{n, m}}\right) .
$$

For $m=1$ and $m \geqslant 2$, we can easily obtain (2.13) and (2.14) by using the formulas (2.11), (2.12) and (2.15), respectively.

\section{Reconstruction of the Coulomb potential function}

Here, we give an explicit formula for the Coulomb potential function by using the nodal lengths. The method used in the proof of the theorem is similar to classical problems: p-Laplacian Sturm-Liouville eigenvalue problem and $p$-Laplacian energy-dependent Sturm-Liouville eigenvalue problem (see [5, 11, $12,18,20,30,31])$.

Theorem 3.1. Let $\mathrm{q}_{\mathrm{m}}^{\mathrm{C}}(\mathrm{x})$ be a real-valued $\mathrm{C}[1, \mathrm{a}]$-function. Then

$$
\mathrm{q}_{\mathrm{m}}^{\mathrm{C}}(x)=\lim _{n \rightarrow \infty} p \delta^{p-1} \lambda_{n, m}\left(\frac{\tilde{\delta} \lambda_{n, m}^{1 / p} l_{j, m}^{n}}{\hat{\pi}}-1\right)
$$

for $j=j_{n}(x)=\max \left\{j: x_{j, m}^{n}<x\right\}$ and $m \in \mathbb{Z}^{+}$.

Proof. We need to consider Theorem 2.4 for the proof. From (2.15), we have

$$
\frac{p \delta^{p} \lambda_{n, m}^{1 / p+1}}{\hat{\pi}} l_{j, m}^{n}=p \delta^{p-1} \lambda_{n, m}+\frac{\left(\delta^{p}-1\right) \lambda_{n, m}^{\frac{1}{p}+1}}{\hat{\pi}} l_{j, m}^{n}+\frac{\lambda_{n, m}^{1 / p}}{\hat{\pi}} \int_{x_{j, m}^{n}}^{x_{j+1, m}^{n}} q_{m}^{C}(t) d t
$$




$$
\begin{aligned}
& +\frac{p\left(\delta^{p}-1\right) \lambda_{n, m}^{\frac{1}{p}+1}}{\hat{\pi}} \int_{x_{j, m}^{n}}^{x_{j+1, m}^{n}}\left(S_{p}^{p}-\frac{1}{p}\right) d t \\
& +\frac{p \lambda_{n, m}^{1 / p}}{\hat{\pi}} \int_{x_{j, m}^{n}}^{x_{j+1, m}^{n}} q_{m}^{C}(t)\left(S_{p}^{p}-\frac{1}{p}\right) d t .
\end{aligned}
$$

Then, we can use similar procedure as those in [20] for $j=j_{n}(x)=\max \left\{j: x_{j, m}^{n}<x\right\}$ to show that

$$
\frac{\lambda_{n, m}^{1 / p}}{\hat{\pi}} \int_{x_{j, m}^{n}}^{x_{j+1, m}^{n}} q_{m}^{C}(t) d t \rightarrow q_{m}^{C}(x)
$$

and

$$
\frac{p\left(\delta^{p}-1\right) \lambda_{n, m}^{\frac{1}{p}+1}}{\hat{\pi}} \int_{x_{j, m}^{n}}^{x_{j+1, m}^{n}}\left(S_{p}^{p}-\frac{1}{p}\right) d t \rightarrow 0, \quad \frac{p \lambda_{n, m}^{1 / p}}{\hat{\pi}} \int_{x_{j, m}^{n}}^{x_{j+1, m}^{n}} q_{m}(t)\left(S_{p}^{p}-\frac{1}{p}\right) d t \rightarrow 0,
$$

pointwise almost everywhere. Hence, we get

$$
\mathrm{q}_{\mathrm{m}}^{\mathrm{C}}(x)=\lim _{n \rightarrow \infty} p \delta^{\mathrm{p}-1} \lambda_{n, m}\left(\frac{\tilde{\delta} \lambda_{n, m}^{1 / p} l_{j, m}^{n}}{\hat{\pi}}-1\right) \text {. }
$$

Theorem 3.2. Let $\left\{l_{j, m}^{n}: j=1,2, \cdots, n-1\right\}_{n=2}^{\infty}$ be a set of nodal lengths of problem (1.1) and (1.2) where $\mathrm{q}_{m}^{\mathrm{C}}$ is a real-valued $\mathrm{C}[1, \mathrm{a}]$-function. Let us define

$$
F_{n, 1}(x)=\frac{p \delta^{p-1}(n \hat{\pi})^{p}}{\tilde{\delta}^{p}(a-1)^{p}}\left(\frac{n l_{j, 1}^{n}}{a-1}-1\right)-\frac{p \delta^{p}(n \hat{\pi})^{p / 2}}{b_{1} \tilde{\delta}^{\frac{p}{2}+1}(a-1)^{\frac{p}{2}+1}}+\frac{1}{\tilde{\delta}(a-1)} \int_{1}^{a} q_{1}^{C}(t) d t,
$$

and

$$
\begin{aligned}
F_{n, m}(x)= & \frac{p \delta^{p-1}(n \hat{\pi})^{p}}{\tilde{\delta}^{p}(a-1)^{p}}\left(\frac{n l_{j, m}^{n}}{a-1}-1\right)-\frac{p \delta^{p}(a-1)^{\frac{m p-2 p-2}{2}}(n \hat{\pi})^{p / 2}}{\tilde{\delta}^{\frac{m p-2 p-2}{2}}\left[b_{1}(a-1)^{\frac{m p-p}{2}}+\cdots+b_{m}(n \hat{\pi})^{\frac{m p-p}{2}}\right]} \\
& +\frac{1}{\tilde{\delta}(a-1)} \int_{1}^{a} q_{m}^{c}(t) d t
\end{aligned}
$$

for $m=1$ and $m \geqslant 2$, respectively. Then, $\left\{F_{n, m}(x)\right\}$ converges to $q_{m}$ pointwise almost everywhere in $L^{1}(1, a)$, for all cases.

Proof. For $m=1$, by the asymptotic formula of eigenvalues (2.3) and nodal lengths (2.13), we get

$$
\begin{aligned}
p \delta^{p-1} \lambda_{n, 1}\left(\frac{\tilde{\delta} \lambda_{n, 1}^{1 / p} l_{j, 1}^{n}}{\hat{\pi}}-1\right)= & p \delta^{p-1} \lambda_{n, 1}\left(\frac{n l_{j, 1}^{n}}{a-1}-1\right)-\frac{p \delta^{p} n^{\frac{p+2}{2}} \hat{\pi}^{\frac{p}{2}}}{b_{1} \tilde{\delta}^{\frac{p}{2}+1}(a-1)^{\frac{p}{2}+1}} l_{j, 1}^{n} \\
& +\frac{n l_{j, 1}^{n}}{\tilde{\delta}(a-1)^{2}} \int_{1}^{a} q_{1}^{c}(t) d t+o(1) .
\end{aligned}
$$


Considering $n l_{j, 1}^{n}=(a-1)+o(1)$, as $n \rightarrow \infty$, we have

$$
\frac{p \delta^{p-1}(n \hat{\pi})^{p}}{\tilde{\delta}^{p}(a-1)^{p}}\left(\frac{n l_{j, 1}^{n}}{a-1}-1\right)-\frac{p \delta^{p}(n \hat{\pi})^{p / 2}}{b_{1} \tilde{\delta}^{\frac{p}{2}+1}(a-1)^{\frac{p}{2}+1}} \rightarrow q_{1}^{C}(x)-\frac{1}{\tilde{\delta}(a-1)} \int_{1}^{a} q_{1}^{C}(t) d t,
$$

pointwise convergence almost everywhere in $\mathrm{L}^{1}(1, a)$.

Similarly, for $m \geqslant 2$, by the asymptotic formula of eigenvalues (2.4) and nodal lengths (2.14), we obtain

$$
\begin{aligned}
p \delta^{p-1} \lambda_{n, m}\left(\frac{\tilde{\delta} \lambda_{n, m}^{1 / p} l_{j, m}^{n}}{\hat{\pi}}-1\right)= & p \delta^{p-1} \lambda_{n, m}\left(\frac{n l_{j, m}^{n}}{a-1}-1\right) \\
& -\frac{p \delta^{p}(a-1)^{\frac{m p-2 p-4}{2}} n^{\frac{p+2}{2}} \hat{\pi}^{\frac{p}{2}}}{\tilde{\delta}^{\frac{m p-2 p-2}{2}}\left[b_{1}(a-1)^{\frac{m p-p}{2}}+\cdots+b_{m} n^{\frac{m p-p}{2}} \hat{\pi}^{\frac{m p}{2}}\right]} l_{j, m}^{n} \\
& +\frac{n l_{j, m}^{n}}{\tilde{\delta}(a-1)^{2}} \int_{1}^{a} q_{m}^{C}(t) d t+o(1) .
\end{aligned}
$$

Considering $n l_{j, m}^{n}=(a-1)+o(1)$, as $n \rightarrow \infty$, we have

$$
\frac{p \delta^{p-1}(n \hat{\pi})^{p}}{\tilde{\delta}^{p}(a-1)^{p}}\left(\frac{n l_{j, m}^{n}}{a-1}-1\right)-\frac{p \delta^{p}(a-1)^{\frac{m p-2 p-2}{2}}(n \hat{\pi})^{p / 2}}{\tilde{\delta} \frac{m p-2 p-2}{2}\left[b_{1}(a-1)^{\frac{m p-p}{2}}+\cdots+b_{m}(n \hat{\pi})^{\frac{m p-p}{2}}\right]} \rightarrow q_{m}^{C}(x)-\frac{1}{\tilde{\delta}(a-1)} \int_{1}^{a} q_{m}^{C}(t) d t,
$$

pointwise convergence almost everywhere in $\mathrm{L}^{1}(1, \mathrm{a})$.

\section{Conclusion}

Generalization of a given problem is an important issue in mathematics. We extend classical SturmLiouville eigenvalue problem with Coulomb potential from two perspectives. Firstly, we define pLaplacian form of Sturm-Liouville equation. On the other hand, we modificate the boundary condition by putting a more general polynomially spectral parameter. In this way, we get more general results for eigenvalues, nodal parameters and Coulomb potential function of the problem (1.1) and (1.2).

\section{References}

[1] V. Ambarzumian, Über eine frage der eigenwerttheorie, Z. Phys., 53 (1929), 690-695. 1

[2] R. K. Amirov, Y. Cakmak, S. Gulyaz, Boundary value problem for second-order differential equations with Coulomb singularity on a finite interval, Indian J. Pure Appl. Math., 37 (2006), 125-140. 1

[3] R. K. Amirov, N. Topsakal, A representation for solutions of Sturm-Liouville equations with Coulomb potential inside finite interval, J. Cumhuriyet Univ. Nat. Sci., 28 (2007), 11-38.

[4] R. K. Amirov, N. Topsakal, Sturm-Liouville operators with Coulomb potential which have discontinuity conditions inside an interval, Integral Transforms Spec. Funct., 19 (2008),923-937. 1

[5] P. Binding, P. Drábek, Sturm-Liouville theory for the p-Laplacian, Studia Sci. Math. Hungar., 40 (2003), 373-396. 1, 3

[6] G. Borg, Eine Umkehrung der Sturm-Liouvilleschen Eigenwertaufgabe, Bestimmung der Differentialgleichung durch die Eigenwerte, (German) Acta Math., 78 (1945), 1-96. 1

[7] P. J. Browne, B. D. Sleeman, Inverse nodal problems for Sturm-Liouville equations with eigenparameter dependent boundary conditions, Inverse Problems, 12 (1996), 377-381. 1

[8] S. A. Buterin, On inverse spectral problem for non-selfadjoint Sturm-Liouville operator on a finite interval, J. Math. Anal. Appl., 335 (2007), 739-749. 1

[9] R. Carlson, Inverse spectral theory for some singular Sturm-Liouville problems, J. Differential Equations, 106 (1993), 121-140. 1

[10] R. Carlson, An inverse spectral problem for Sturm-Liouville operators with discontinuous coefficients, Proc. Amer. Math. Soc., 120 (1994), 475-484. 1 
[11] H. Y. Chen, On generalized trigonometric functions, Master of Science thesis, National Sun Yat-sen University, Kaohsiung, Taiwan, (2009). 1, 1, 1.1, 3

[12] Á. Elbert, On the half-linear second order differential equations, Acta Math. Hungar., 49 (1987), 487-508. 1, 3

[13] G. Freiling, V. A. Yurko, Inverse problems for Sturm-Liouville equations with boundary conditions polynomially dependent on the spectral parameter, Inverse Problems, 26 (2010), 17 pages. 1

[14] N. J. Guliyev, Inverse eigenvalue problems for Sturm-Liouville equations with spectral parameter linearly contained in one of the boundary conditions, Inverse Problems, 21 (2005), 1315-1330. 1

[15] T. Gulsen, E. Yilmaz, Inverse nodal problem for p-Laplacian diffusion equation with polynomially dependent spectral parameter, Commun. Fac. Sci. Univ. Ank. Sér. A1 Math. Stat., 65 (2016), 23-36. 1

[16] O. H. Hald, Discontinuous inverse eigenvalue problems, Comm. Pure Appl. Math., 37 (1984), 539-577. 1

[17] O. H. Hald, J. R. McLaughlin, Solutions of inverse nodal problems, Inverse Problems, 5 (1989), 307-347. 1

[18] H. Koyunbakan, Erratum: Inverse nodal problem for p-Laplacian energy-dependent Sturm-Liouville equation, Bound. Value Probl., 2014 (2014), 2 pages. 1, 3

[19] H. Koyunbakan, E. Yilmaz, Reconstruction of the potential function and its derivatives for the diffusion operator, Z. Naturforsch. A, 63 (2008), 127-130. 1

[20] C. K. Law, W.-C. Lian, W.-C. Wang, The inverse nodal problem and the Ambarzumyan problem for the p-Laplacian, Proc. Roy. Soc. Edinburgh Sect. A, 139 (2011), 1261-1273. 1, 3, 3

[21] C. K. Law, C.-F. Yang, Reconstructing the potential function and its derivatives using nodal data, Inverse Problems, 14 (1998), 299-312. 1

[22] N. Levinson, The inverse Sturm-Liouville problem, Mat. Tidsskr. B., 1949 (1949), 25-30.

[23] B. M. Levitan, On the determination of the Sturm-Liouville operator from one and two spectra, Math. USSR Izv., 12 (1978), 179-193.

[24] V. A. Marchenko, Sturm-Liouville operators and applications, Translated from the Russian by A. Iacob, Operator Theory: Advances and Applications, Birkhäuser Verlag, Basel, (1986). 1

[25] J. R. McLaughlin, Inverse spectral theory using nodal points as data-a uniqueness result, J. Differential Equations, 73 (1988), 354-362. 1

[26] A. S. Ozkan, B. Keskin, Inverse nodal problems for Sturm-Liouville equation with eigenparameter-dependent boundary and jump conditions, Inverse Probl. Sci. Eng., 23 (2015), 1306-1312. 1

[27] W. Y. Ping, C. T. Shieh, Inverse problems for Sturm-Liouville equations with boundary conditions linearly dependent on the spectral parameter from partial information, Results Math., 65 (2014), 105-119. 1

[28] J. Pöschel, E. Trubowitz, Inverse spectral theory, Pure and Applied Mathematics, Academic Press, Inc., Boston, MA, (1987). 1

[29] J. Walter, Regular eigenvalue problems with eigenvalue parameter in the boundary condition, Math. Z., 133 (1973), 301312. 1

[30] W.-C. Wang, Direct and inverse problems for one dimensional p-Laplacian operators, PhD thesis, National Sun Yat-sen University, Taiwan, (2010). 1, 3

[31] W. C. Wang, Y. H. Cheng, W. C. Lian, Inverse nodal problems for the p-Laplacian with eigenparameter dependent boundary conditions, Math. Comput. Modelling, 54 (2011), 2718-2724. 1, 2.1, 3

[32] C.-F. Yang, X.-P. Yang, Ambarzumyan's theorem with eigenparameter in the boundary conditions, Acta Math. Sci. Ser. B Engl. Ed., 31 (2011), 1561-1568. 1

[33] C.-F. Yang, X.-P. Yang, Inverse nodal problems for the Sturm-Liouville equation with polynomially dependent on the eigenparameter, Inverse Probl. Sci. Eng., 19 (2011), 951-961. 1

[34] A. Yantır, Oscillation theory for second order differential equations and dynamic equations on time scales, Master of Science thesis, Izmir institue of Technology, Izmir, Turkey, (2004). 2

[35] V. A. Yurko, Inverse spectral problems for differential operators and their applications, Analytical Methods and Special Functions, Gordon and Breach Science Publishers, Amsterdam, (2000). 1

[36] V. A. Yurko, Inverse nodal problems for Sturm-Liouville operators on star-type graphs, J. Inverse Ill-Posed Probl., 16 (2008), 715-722. 1 